SOX2 ... senses bacterial DNA in a sequencespecific manner

\title{
New sensor of bacterial DNA
}

The transcription factor SOX2 is known for its role in the maintenance of embryonic and neural stem cells. Now, reporting in Nature Immunology, Xia et al. show that SOX2 is also expressed by neutrophils, where it functions as a sensor of cytosolic bacterial DNA.

When Xia et al. investigated the expression pattern of SOX 2 in haematopoietic stem cells in mouse bone marrow, they found SOX $2^{+}$cells with a morphology similar to neutrophils. Indeed, these cells expressed neutrophil markers, and among all mature haematopoietic cells tested, only peripheral neutrophils expressed SOX2 at both the mRNA and protein level. SOX2 was constitutively expressed in the cytoplasm of peripheral neutrophils from both mice and humans.

To examine the role of SOX 2 in neutrophils, the authors generated mice with a conditional deletion of
Sox2 in these cells. These mice were more sensitive to infection with Listeria monocytogenes and other bacterial pathogens than control mice. Furthermore, infected mice with SOX2-deficient neutrophils had decreased concentrations of pro-inflammatory cytokines, such as tumour necrosis factor (TNF), interleukin-6 (IL-6) and IL-1 $\beta$, but the level of type I interferons (IFNs) was unaffected. Of note, neutrophilmediated bacterial clearance was shown to be independent of the cytosolic DNA sensor cGAS and its adaptor stimulator of IFN genes (STING).

SOX2 is a transcription factor that binds well-defined DNA sequences. To test if SOX2 senses and binds bacterial DNA in neutrophils, the authors isolated neutrophils from the Sox 2 conditional knockout mice and transfected them with bacterial genomic DNA; the SOX2-deficient

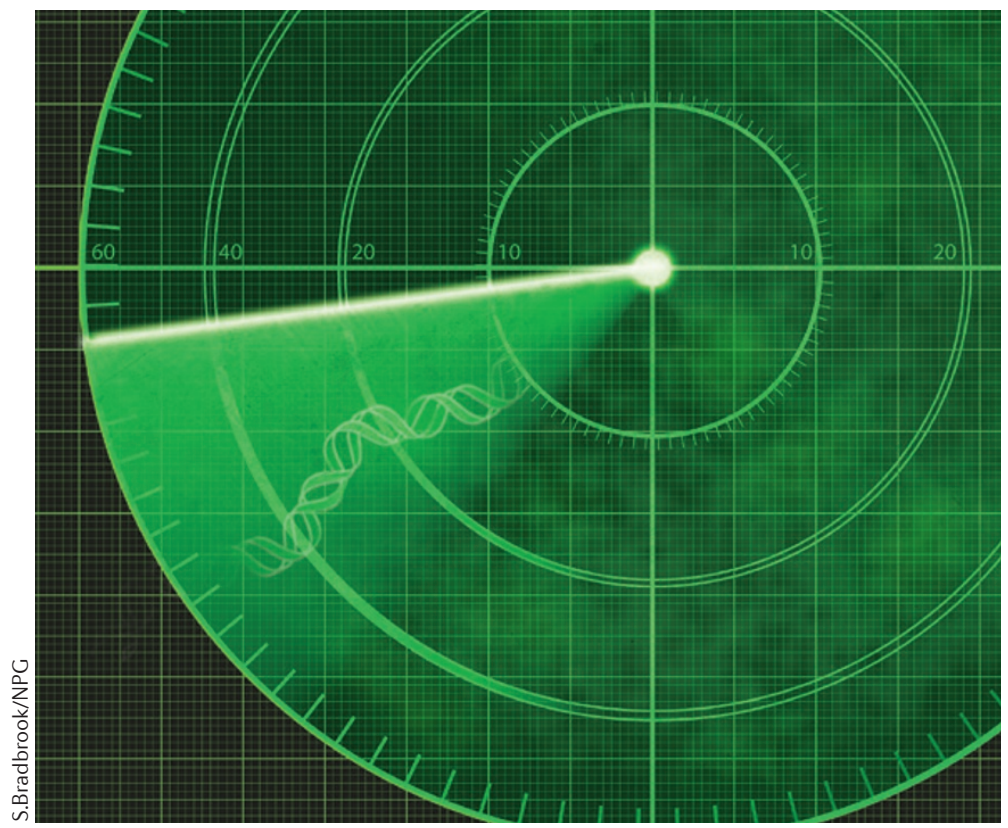

neutrophils produced lower levels of TNF, IL- 6 and IL- $1 \beta$ than control mice. Interestingly, sequence analysis showed that several regions of the L. monocytogenes genome matched the canonical SOX2-binding sequence, and SOX2-matched bacterial DNA (but not unmatched L. monocytogenes DNA) bound to SOX2 in neutrophils and induced the production of pro-inflammatory cytokines following transfection. Thus, SOX2 seems to be required for neutrophil activation after challenge with bacterial DNA and it senses bacterial DNA in a sequence-specific manner.

Finally, the authors investigated the mechanisms by which SOX2 senses DNA. Through a series of experiments, they showed that SOX2 associates with TGF $\beta$-activated kinase 1 (TAK1) and TAK1-binding protein 2 (TAB2) after neutrophil stimulation with bacterial DNA. Knockdown of TAK1 or TAB2 in neutrophils decreased activation of the nuclear factor- $\kappa \mathrm{B}(\mathrm{NF}-\kappa \mathrm{B})$ and AP-1 signalling pathways after transfection with L. monocytogenes DNA. Furthermore, mice deficient in TAK1 or TAB2 had higher bacterial loads in the spleen and liver than wild-type mice following infection. Hence, TAK1 and TAB2 are required for the SOX2-mediated bacterial clearance in neutrophils.

In summary, this study reveals a new role for SOX2 in sensing bacterial DNA in the cytoplasm of neutrophils. This could be important for the development of therapeutic strategies for treatment of infectious diseases. Elisabeth Kugelberg

ORIGINAL RESEARCH PAPER Xia, P. et al. Sox2 functions as a sequence-specific DNA sensor in neutrophils to initiate innate immunity against microbial infection. Nature Immunol. http://dx.doi. org/10.1038/ni.3117 (2015) 